\title{
Complex Excited State Polarizabilities in the ADC/ISR Framework
}

Maximilian Scheurer, ${ }^{1, \text { a) }}$ Thomas Fransson, ${ }^{1,2}$ Patrick Norman, ${ }^{3}$ Andreas Dreuw, ${ }^{1}$ and

Dirk R. Rehn ${ }^{1, b)}$

1) Interdisciplinary Center for Scientific Computing, Ruprecht-Karls University,

Im Neuenheimer Feld 205, D-69120 Heidelberg, Germany

2)Department of Physics, AlbaNova University Center, Stockholm University,

Stockholm SE-106 91, Sweden

${ }^{3)}$ Department of Theoretical Chemistry and Biology, School of

Engineering Sciences in Chemistry, Biotechnology and Health,

KTH Royal Institute of Technology, SE-106 91 Stockholm,

Sweden

(Dated: 13 May 2020)

We present the derivation and implementation of complex, frequency-dependent polarizabilities for excited states using the algebraic-diagrammatic construction for the polarization propagator (ADC) and its intermediate state representation (ISR). Based on the complex polarizability we evaluate $\mathrm{C}_{6}$ dispersion coefficients for excited states. The methodology is implemented up to third order in perturbation theory in the Python-driven adcc toolkit for the development and application of ADC methods. We exemplify the approach using small model systems and compare it to results from coupled-cluster theory and from experiments.

\footnotetext{
a) Electronic mail: maximilian.scheurer@iwr.uni-heidelberg.de
}

b)Electronic mail: rehn@uni-heidelberg.de 


\section{INTRODUCTION}

Response theory offers a framework to derive and compute a multitude of molecular properties. ${ }^{1,2}$ Through its general formulation it can be applied to both density functional theory (DFT) and $a b$-initio methods. Within the algebraic-diagrammatic construction scheme for the polarization propagator $(\mathrm{ADC}),{ }^{3}$ such response properties can elegantly and easily be derived owing to its Hermitian formulation using the formalism of the intermediate state representation (ISR). ${ }^{4,5}$ The latter offers direct access to excited state wave functions and operators, which makes it straightforward to implement method-independent spectral representations of molecular response functions. This strategy has been successfully employed to evaluate several response properties with ADC, e.g., static and frequency-dependent polarizabilities of the electronic ground state, ${ }^{5-7}$ two-photon absorption, ${ }^{8}$ and resonant inelastic X-ray scattering cross sections. ${ }^{9}$ As a matter of fact, only minor programming effort is needed once the required building blocks of the ISR are in place. Here, we expand upon previous work by addressing frequency-dependent electric dipole polarizabilities for electronically excited states within the ADC/ISR framework. These can be evaluated from either the excited state linear response function or the double residue of the ground state cubic response function. ${ }^{10-12}$ Following the former route via damped response theory ${ }^{2}$ offers access to one-photon absorption cross-sections as well as $C_{6}$ dispersion coefficients. We have implemented a protocol for the calculation of these properties through third order of perturbation theory in our recently published Python-driven toolkit for ADC method development, adcc, ${ }^{13}$ which made the implementation particularly straightforward.

Over the last decades, numerous quantum chemical methods have been employed for the calculation of frequency-dependent excited state polarizabilities, including wave function methods such as Hartree-Fock, ${ }^{10,14-16}$ coupled-cluster, ${ }^{11,12,17-21}$ and multi-reference approaches, ${ }^{15,16,18,22}$ as well as DFT methods. ${ }^{20,23-25}$ Calculations of excited state $C_{6}$ dispersion coefficients are more scarce $^{24}$ and this property is also difficult to determine experimentally. Generally, two possible approaches to calculate molecular properties exist: either by derivatives of the energy, or through expectation values. A comparative analysis of these approaches has recently been conducted for ADC methods. ${ }^{26}$ For excited state polarizabilities, both approaches have been reported for equation-of-motion coupled-cluster with singles and doubles (EOM-CCSD). ${ }^{12,21,27,28}$ The ISRbased ansatz described in this work is comparable to the expectation-value coupled-cluster approach to molecular properties, and both methods will be analyzed and compared to experimental 
data where available.

The remainder of this paper is structured as follows. First, we briefly outline the theoretical derivation and implementation. Second, the employed computational methodology is presented, followed by the results of test calculations on $s$-tetrazine, pyrimidine, formaldehyde, naphthalene, uracil, and $p$-nitroaniline. Finally, a brief outlook for future applications using the presented methodology is given.

\section{THEORETICAL DERIVATION AND IMPLEMENTATION}

The theoretical background of ADC and the ISR formalism has already been presented in great detail. ${ }^{3,4,29,30}$ Here, we will just briefly outline the necessary building blocks for our approach to excited state polarizabilities. Most important is the Hermitian eigenvalue problem $\mathbf{M Y}=\mathbf{Y} \Omega$, involving the ADC matrix $\mathbf{M}$, the matrix of eigenvectors $\mathbf{Y}=\left\{\mathbf{y}_{n}\right\}$, and the matrix $\Omega$ with excitation energies $\omega_{n}$ on the diagonal. The ADC matrix corresponds to the matrix representation of the shifted Hamiltonian, $M_{I J}=\left\langle\tilde{\Psi}_{I}\left|\hat{H}-E_{0}\right| \tilde{\Psi}_{J}\right\rangle$, in the basis of intermediate states $\left\{\left|\tilde{\Psi}_{J}\right\rangle\right\}$. A key feature, which we can exploit for the derivation of molecular response properties, is the IS expansion of an excited state wave function, given by $\left|\Psi_{n}\right\rangle=\sum_{J} Y_{J n}\left|\tilde{\Psi}_{J}\right\rangle$. This IS formalism also allows one to express other operators in the same basis. For example, a general one-particle operator, $\hat{d}=\sum_{p q} d_{p q} c_{p}^{\dagger} c_{q}$ can be represented in the IS basis as

$$
B_{I J}=\left\langle\tilde{\Psi}_{I}|\hat{d}| \tilde{\Psi}_{J}\right\rangle-\delta_{I J}\left\langle\Psi_{0}|\hat{d}| \Psi_{0}\right\rangle
$$

where the matrix is again shifted by the ground state expectation value on the diagonal. With the same "recipe", the so-called modified transition moments from ground to excited states can be formulated as $F_{J}(\hat{d})=\left\langle\tilde{\Psi}_{J}|\hat{d}| \Psi_{0}\right\rangle$. Further details and discussion of these ISR equations can be found in Ref. 4. The quantities introduced above suffice to conveniently express various groundstate response functions in the ADC/ISR formalism. To extend this approach to the excited state, we introduce the modified quantities: $\mathbf{M}_{f}=\mathbf{M}-\mathbf{1} \omega_{f}-\omega_{f} \mathbf{y}_{f} \mathbf{y}_{f}^{\dagger}$, which shifts the diagonal by $\omega_{f}$ and projects the $f$-th eigenstate out of the matrix $\mathbf{M}$, and the analogously modified ISR operator matrix $\mathbf{B}_{f}=\mathbf{B}-\omega_{f} \mathbf{y}_{f} \mathbf{y}_{f}^{\dagger}$, which replace the original ADC matrix $\mathbf{M}$ and operator matrix $\mathbf{B}$ in the corresponding excited state response function.

From time-dependent perturbation theory, the electric dipole polarizability of an electronic state 
$\left|\Psi_{N}\right\rangle$ can be obtained as a frequency-dependent response function ${ }^{5}$

$$
\begin{aligned}
\alpha_{A B}^{N}(\omega)= & -\left\langle\Psi_{N}\left|\hat{\mu}_{A}\left(\hbar \omega-\hat{H}+E_{N}\right)^{-1} \hat{\mu}_{B}\right| \Psi_{N}\right\rangle \\
& +\left\langle\Psi_{N}\left|\hat{\mu}_{B}\left(\hbar \omega+\hat{H}-E_{N}\right)^{-1} \hat{\mu}_{A}\right| \Psi_{N}\right\rangle,
\end{aligned}
$$

with the dipole operator $\hat{\mu}$ and the respective Cartesian component $A$ or $B$. This expression is valid both for the electronic ground state $|0\rangle$ and for electronically excited states $|f\rangle$. Equation (2) can be recast to the so-called sum-over-states (SOS) expression or spectral representation to yield the polarizability of an excited state $f$ as

$$
\alpha_{A B}^{f}(\omega)=\frac{1}{\hbar} \sum_{n \neq f}\left[\frac{\left\langle f\left|\hat{\mu}_{A}\right| n\right\rangle\left\langle n\left|\hat{\mu}_{B}\right| f\right\rangle}{\omega_{n}-\omega_{f}-\omega-i \gamma_{n}}+\frac{\left\langle f\left|\hat{\mu}_{B}\right| n\right\rangle\left\langle n\left|\hat{\mu}_{A}\right| f\right\rangle}{\omega_{n}-\omega_{f}+\omega+i \gamma_{n}}\right] .
$$

The response function from eq (2) was further made resonant-convergent by introducing $\gamma_{n}$, which is related to the inverse, finite life time of excited state $|n\rangle$. We have used a common damping parameter $\gamma_{n}=\gamma$ for every state, ${ }^{9}$ but it could easily be made state-specific in the implementation. Note that the prefactor $\frac{1}{\hbar}$ is omitted in the following. To arrive at a programmable expression, we substitute the transition moments with their corresponding ISR form

$$
\begin{aligned}
\alpha_{A B}^{f}(\omega)=\sum_{\substack{n \neq f, n \neq 0}}\left[\frac{\mathbf{y}_{f}^{\dagger} \mathbf{B}_{f}^{A} \mathbf{y}_{n} \mathbf{y}_{n}^{\dagger} \mathbf{B}_{f}^{B} \mathbf{y}_{f}}{\omega_{n}-\omega_{f}-\omega-i \gamma}+\frac{\mathbf{y}_{f}^{\dagger} \mathbf{B}_{f}^{B} \mathbf{y}_{f} \mathbf{y}_{f}^{\dagger} \mathbf{B}_{f}^{A} \mathbf{y}_{f}}{\omega_{n}-\omega_{f}+\omega+i \gamma}\right] \\
+\frac{\mathbf{y}_{f}^{\dagger} F^{A} F^{B \dagger} \mathbf{y}_{f}}{-\omega_{f}-\omega-i \gamma}+\frac{\mathbf{y}_{f}^{\dagger} F^{B} F^{A \dagger} \mathbf{y}_{f}}{-\omega_{f}+\omega+i \gamma} .
\end{aligned}
$$

This equation contains the modified ISR matrix $\mathbf{B}_{f}$, which does not couple to state $f$. The last two terms describe the coupling to the electronic ground state which is not accounted for in the $\mathbf{B}_{f}$ matrix. Finally, we replace the summation over $n$ with the ISR as well ${ }^{5}$ and find

$$
\begin{aligned}
\alpha_{A B}^{f}(\omega) & =\mathbf{y}_{f}^{\dagger} \mathbf{B}_{f}^{A}\left(\mathbf{M}_{f}-\omega-i \gamma\right)^{-1} \mathbf{B}_{f}^{B} \mathbf{y}_{f}+\mathbf{y}_{f}^{\dagger} \mathbf{B}_{f}^{B}\left(\mathbf{M}_{f}+\omega+i \gamma\right)^{-1} \mathbf{B}_{f}^{A} \mathbf{y}_{f} \\
& +\frac{\mathbf{y}_{f}^{\dagger} F^{A} F^{B^{\dagger}} \mathbf{y}_{f}}{-\omega_{f}-\omega-i \gamma}+\frac{\mathbf{y}_{f}^{\dagger} F^{B} F^{A \dagger} \mathbf{y}_{f}}{-\omega_{f}+\omega+i \gamma} .
\end{aligned}
$$

Instead of full matrix inversion, a system of linear equations is solved, which for the first term corresponds to

$$
\left(\mathbf{M}_{f}-\omega-i \gamma\right) \mathbf{x}_{f}=\mathbf{B}_{f} \mathbf{y}_{f}
$$


yielding the response vector $\mathbf{x}_{f}$. Subsequently, the polarizability can be evaluated as

$$
\begin{aligned}
\alpha_{A B}^{f}(\omega) & =\mathbf{y}_{f}^{\dagger} \mathbf{B}_{f}^{A} \mathbf{x}_{f}^{B}+\mathbf{y}_{f}^{\dagger} \mathbf{B}_{f}^{B} \mathbf{x}_{f}^{A} \\
& +\frac{\mathbf{y}_{f}^{\dagger} F^{A} F^{B \dagger} \mathbf{y}_{f}}{-\omega_{f}-\omega-i \gamma}+\frac{\mathbf{y}_{f}^{\dagger} F^{B} F^{A \dagger} \mathbf{y}_{f}}{-\omega_{f}+\omega+i \gamma}
\end{aligned}
$$

From the complex, frequency-dependent polarizability, $C_{6}$ dispersion coefficients can be obtained as described in Ref. 6.

\section{A. Implementation}

Complex algebra is avoided by recasting the equation to a double-dimensional form

$$
\left(\begin{array}{cc}
\mathbf{M}_{f}-\mathbf{1} \omega & \mathbf{1} \gamma \\
\mathbf{1} \gamma & -\mathbf{M}_{f}+\mathbf{1} \omega
\end{array}\right)\left(\begin{array}{c}
\mathbf{x}_{R} \\
\mathbf{x}_{I}
\end{array}\right)=\left(\begin{array}{c}
\mathbf{B}_{f} \mathbf{y}_{f} \\
0
\end{array}\right)
$$

where the solution vector contains a real and an imaginary block $\mathbf{x}_{R}$ and $\mathbf{x}_{I}$, respectively. The right-hand side is treated in the same manner, where the imaginary part is zero for real-valued operators.

Only the matrix-vector products of the un-shifted ADC/ISR matrices with a trial vector $\mathbf{r}$ are available in our code and the modified matrices $\mathbf{M}_{f}$ and $\mathbf{B}_{f}$ are implemented by projecting out all components along the eigenvector $\mathbf{x}_{f}$ after the matrix multiplication, i.e.,

$$
\mathbf{M}_{f} \mathbf{r}=\mathbf{M r}-\omega_{f} \mathbf{r}-\mathbf{x}_{f} \frac{\mathbf{x}_{f}^{\dagger} \mathbf{M r}}{\mathbf{x}_{f}^{\dagger} \mathbf{x}_{f}} .
$$

We employ a conjugate gradient (CG) algorithm $^{31}$ with a Jacobi preconditioner to solve eq (8) for a given right-hand side. For the static polarizability, the problem in eq (8) reduces to

$$
\mathbf{M}_{f} \mathbf{x}_{R}=\mathbf{B}_{f} \mathbf{y}_{f}
$$

which we solve using a standard Jacobi algorithm including Anderson mixing (sometimes called DIIS mixing). ${ }^{32}$ The implementation was achieved using our recently published adcc toolkit. ${ }^{13}$ All additional working equations (e.g., the $\mathbf{B}$ matrix) were implemented on the $\mathrm{C}++$ layer and are conveniently exposed to the Python layer. Since only the full matrix representation for $\mathbf{B}$ is given in Ref. 4, the necessary matrix-vector product was derived and the programmable expressions are shown in Appendix A. Iterative solvers and evaluation of the final polarizability expressions 
are written entirely in Python. For completeness, complex frequency-dependent polarizabilities were implemented as well. ${ }^{6}$ To test our implementation, eq (3) was evaluated for small test systems $\left(\mathrm{H}_{2} \mathrm{O} / 6-31 \mathrm{G}, \mathrm{LiH} / \mathrm{STO}-3 \mathrm{G}\right)$, where a full ADC(2) matrix diagonalization is easily achievable. The results from evaluating the SOS expression were then compared to the result from the linear solvers, and were found to agree, which confirmed the validity of our implementation (data not shown).

\section{COMPUTATIONAL DETAILS}

Geometries for $s$-tetrazine, pyrimidine, uracil and $p$-nitroaniline (PNA) were obtained from Ref. 12. For $s$-tetrazine, static polarizabilities of the ground state and the $1^{1} \mathrm{~B}_{1 \mathrm{u}}$ excited state were computed using the Sadlej-pVTZ basis set $^{33}$ and the geometry of the corresponding electronic state, as described in Ref. 12. Results were obtained using ADC(2), ADC(2)-x, and ADC(3), as now implemented in adcc. SCF results were obtained using pyscf. ${ }^{34,35}$ In all ADC calculations, the second-order ISR was employed. ${ }^{4,6,26}$ In combination with third-order ADC, this results in the $\mathrm{ADC}(3 / 2)$ approximation which is referred to as $\mathrm{ADC}(3)$ throughout this paper. For consistency, the calculations using the EOM-CCSD derivative and expectation-value approaches were repeated from Ref. 12, employing the Q-Chem 5.2 program package. ${ }^{36}$ The same methods were employed to compute static polarizabilities of the pyrimidine ground state and the $1^{1} \mathrm{~B}_{2}$ excited state. Formaldehyde and naphthalene were optimized at the MP(2)/cc-pVTZ level of theory using Q-Chem $5.2,{ }^{36}$ where the former was placed in the $x z$-plane and the latter in the $x y$-plane. For formaldehyde, the polarizabilities of the ground state and the $1^{1} \mathrm{~B}_{1}$ excited state were computed, whereas the ground state and $1^{1} \mathrm{~B}_{3 \mathrm{u}}$ state polarizabilities were obtained for naphthalene, using all three ADC methods and CCSD with the aug-cc-pVDZ basis set. For uracil and PNA, the aug-ccpVDZ basis set was employed to compute the polarizabilities of the ground state and $1^{1} \mathrm{~A}^{\prime \prime}$ and $2^{1} \mathrm{~A}^{\prime}$, as well as $2^{1} \mathrm{~A}_{1}$ excited states, respectively. $\mathrm{C}_{6}$ dispersion coefficients of all states were computed according to the procedure outlined in Ref. 6, using all ADC methods for $s$-tetrazine and pyrimidine, and $\mathrm{ADC}(2)$ only for uracil and PNA. Results were analyzed using $\mathrm{cclib}^{37}$, pandas $^{38,39}$, and plotted using matplotlib ${ }^{40}$ and seaborn. ${ }^{41}$ 


\section{NUMERICAL CASE STUDIES}

To illustrate our implementation of excited state polarizabilities and $C_{6}$ coefficients, we have computed these properties for $s$-tetrazine, pyrimidine, uracil, and PNA. The static polarizabilities of these molecules were previously investigated using EOM-CCSD. ${ }^{12}$ We compare our ADC results with this study, analysing the ADC/ISR results at different levels of perturbation theory, contrasted to EOM-CCSD results using a derivative or expectation-value scheme. In addition, static polarizabilities of formaldehyde and naphthalene are compared to experimental data and among the employed methodologies. Anticipated trends for excited state polarizabilities have been thoroughly discussed in Ref. 12: In brief, states with a large exciton size tend to have larger polarizabilities than the electronic ground state (e.g., Rydberg states), whereas the opposite should be the case for excited states with a large permanent dipole moment, e.g., chargetransfer (CT) states. From the SOS expression of the polarizability (eq (3)), it also becomes clear that low-lying dipole-allowed excited states should possess larger polarizabilities than the elec-

tronic ground state due to coupling to the ground state and to higher-lying excited states. ${ }^{12}$ To discuss the different computational methods, we use absolute differences of isotropic polarizabilities $\left(\alpha_{\mathrm{iso}}=\frac{1}{3}\left(\alpha_{x x}+\alpha_{y y}+\alpha_{z z}\right)\right)$. Since derivative-based CCSD (CCSD Der.) is most different from all other methods, which are expectation-value-based, we use its results as a reference and compute the deviation as $\delta_{\text {Der. }}=\mid \alpha_{\text {iso }}($ CCSD Der. $)-\alpha_{\text {iso }}($ expectation-value method $) \mid$.

\section{A. $s$-Tetrazine and Pyrimidine}

Table I shows the Cartesian components of the static polarizabilities for the ground and $1^{1} \mathrm{~B}_{1 \mathrm{u}}$ states of $s$-tetrazine, as obtained using ADC and CCSD methods. ${ }^{12}$ As previously stated, most CCSD results discussed herein have already been reported, ${ }^{12}$ and have only been amended by CCSD expectation-value (E.V.) results for completeness.

For the ground state static polarizabilities, all methods yield similar results, with differences $\delta_{\text {Der. }}$ of 3.78, 6.86, 2.53, and 1.61 au for $\operatorname{ADC}(2), \operatorname{ADC}(2)-x, \operatorname{ADC}(3)$, and CCSD E.V., respectively. For excited state polarizabilities the differences are larger, with a decreasing discrepancy trend for the ADC hierarchy of 14.91 au for $\operatorname{ADC}(2), 8.58$ au for $\operatorname{ADC}(2)-x$, and 6.64 au for ADC(3). By comparison, the deviation for CCSD E.V. is 11.32 au. As such, we note that ADC(3) is in closest agreement with the derivative-based EOM-CCSD result. An experimental result for 
Complex Excited State Polarizabilities using ADC

TABLE I. Static polarizabilities of the $s$-tetrazine ground and excited $1^{1} \mathrm{~B}_{1 \mathrm{u}}$ states. ${ }^{\mathrm{a}}$

\begin{tabular}{l|c|c|c|c|c}
\hline State & \multicolumn{2}{|c|}{ ground state } & \multicolumn{2}{|c}{$1{ }^{1} \mathrm{~B}_{1 \mathrm{u}}$} & \multicolumn{3}{c}{} \\
\hline & $\left(\alpha_{x x}, \alpha_{y y}, \alpha_{z z}\right)$ & $\alpha_{\text {iso }}$ & $\left(\alpha_{x x}, \alpha_{y y}, \alpha_{z z}\right)$ & $\alpha_{\text {iso }}$ & $E_{\text {exc }}$ \\
\hline ADC(2) & $(66.08,61.26,33.50)$ & 53.61 & $(39.06,78.55,15.71)$ & 44.44 & 2.20 \\
ADC(2)-x & $(69.38,64.96,35.72)$ & 56.69 & $(50.64,80.08,21.60)$ & 50.77 & 1.31 \\
ADC(3) & $(64.10,59.57,33.41)$ & 52.36 & $(57.28,74.94,25.90)$ & 52.70 & 2.18 \\
CCSD Der. & $(60.73,56.02,32.73)$ & 49.83 & $(66.03,80.09,31.93)$ & 59.35 & 2.39 \\
CCSD E.V. & $(62.82,58.01,33.48)$ & 51.44 & $(49.28,71.75,23.05)$ & 48.03 & 2.39 \\
\hline
\end{tabular}

a Polarizability components $\left(\alpha_{A A}, \alpha_{\text {iso }}\right)$ in au, excitation energies $\left(E_{\text {exc }}\right)$ in $\mathrm{eV}$.

the anisotropy of the polarizability $\left(\Delta \alpha=\frac{1}{2}\left(\alpha_{y y}+\alpha_{x x}\right)-\alpha_{z z}\right)$ is reported as 5.4 and 45.2 au for the ground state and the lowest singlet state, respectively. ${ }^{42}$ All computational methods overshoot the anisotropy for the electronic ground state $(30.2,31.5,28.4,25.6$, and 26.9 au for ADC(2), ADC(2)-x, ADC(3), CCSD Der., and CCSD E.V. respectively). However, the anisotropy of the lowest singlet state static polarizability match the experimental result resonably well $(43.1,43.8$, 40.2, 41.1, and 37.5 au for ADC(2), ADC(2)-x, ADC(3), CCSD Der., and CCSD E.V. respectively).

TABLE II. Static polarizabilities of the pyrimidine ground state and $1^{1} \mathrm{~B}_{2}{ }^{\mathrm{a}}$

\begin{tabular}{l|c|c|c|c|c}
\hline State & \multicolumn{2}{|c|}{ ground state } & \multicolumn{2}{|c}{$1^{1} \mathrm{~B}_{2}$} & \multicolumn{3}{l}{} \\
\hline & $\left(\alpha_{x x}, \alpha_{y y}, \alpha_{z z}\right)$ & $\alpha_{\text {iso }}$ & $\left(\alpha_{x x}, \alpha_{y y}, \alpha_{z z}\right)$ & $\alpha_{\text {iso }}$ & $E_{\text {exc }}$ \\
\hline ADC(2) & $(73.48,38.69,76.22)$ & 62.80 & $(118.89,26.65,38.79)$ & 61.45 & 4.32 \\
ADC(2)-x & $(77.21,39.99,79.97)$ & 65.72 & $(114.28,31.22,51.10)$ & 65.53 & 3.44 \\
ADC(3) & $(71.24,38.27,73.61)$ & 61.04 & $(104.45,33.64,57.48)$ & 65.19 & 4.50 \\
CCSD Der. & $(67.79,37.50,70.18)$ & 58.49 & $(111.76,42.13,71.38)$ & 75.09 & 4.59 \\
CCSD E.V. & $(70.05,38.25,72.52)$ & 60.27 & $(102.93,33.16,51.14)$ & 62.41 & 4.59 \\
\hline
\end{tabular}

aPolarizability components $\left(\alpha_{A A}, \alpha_{\text {iso }}\right)$ in au, excitation energies $\left(E_{\text {exc }}\right)$ in $\mathrm{eV}$.

For the pyrimidine molecule, a similar trend is observed for the agreement between computational results, which are shown in Table II. Here, we find small discrepancies $\delta_{\text {Der. }}$ for ground state 
polarizabilities of 4.31, 7.23, 2.55, and 1.78 au for for $\mathrm{ADC}(2), \mathrm{ADC}(2)-\mathrm{x}, \mathrm{ADC}(3)$, and CCSD E.V., respectively. Deviations for the excited state (here $1^{1} \mathrm{~B}_{2}$ ) to CCSD Der. are decreasing from $\operatorname{ADC}(2)(13.65 \mathrm{au}), \operatorname{ADC}(3)(9.90 \mathrm{au})$, to $\operatorname{ADC}(2)-x(9.56 \mathrm{au})$ and the difference to CCSD E.V. lies between $\mathrm{ADC}(3)$ and $\mathrm{ADC}(2)$ (12.68 au). Both excited states are of $n \rightarrow \pi^{*}$ character, and the largest increase in polarizability is found for in-plane components. This is consistent for all ADC methods in comparison to EOM-CCSD. ${ }^{12}$ While all methods based on ISR/expectation values are capable of predicting these trends for $s$-tetrazine and pyrimidine correctly, one notices that trends for out-of-plane components ( $\alpha_{z z}$ and $\alpha_{y y}$ for $s$-tetrazine and pyrimidine, respectively), are not in agreement with the EOM-CCSD derivative approach. However, this observation is made for ADC and CCSD E.V., thus, the effect is solely related to the ansatz to compute the polarizability, and not to the method itself. Note that coupling to the electronic ground state is negligible for the reported polarizabilities, since the $n \rightarrow \pi^{*}$ states are dipole-forbidden. Another observation that requires discussion is the reduced discrepancy to CCSD Der. results with increasing order of perturbation theory from $\operatorname{ADC}(2)$ toward $\operatorname{ADC}(3)$. In a recent study, it has been demonstrated that $\operatorname{ADC}(3)$ yields orbital relaxation effects for $p-h$ excited states through higher order of perturbation theory by including $2 p-2 h$ states. $^{26}$ This explains the trends observed for the employed ADC schemes. Our numerical results thus yield the anticipated behavior of the respective ADC schemes, and provide values comparable to the related CCSD E.V. approach.

\section{B. Formaldehyde and Naphthalene}

TABLE III. Static polarizabilities of the formaldehyde ground state and $1{ }^{1} \mathrm{~B}_{1} \cdot{ }^{\mathrm{a}}$

\begin{tabular}{l|c|c|cc|c}
\hline State & \multicolumn{2}{|c|}{ ground state } & \multicolumn{2}{|c}{$1^{1} \mathrm{~B}_{1}$} \\
\hline & $\left(\alpha_{x x}, \alpha_{y y}, \alpha_{z z}\right)$ & $\alpha_{\text {iso }}$ & $\left(\alpha_{x x}, \alpha_{y y}, \alpha_{z z}\right)$ & $\alpha_{\text {iso }}$ & $E_{\text {exc }}$ \\
\hline ADC(2) & $(17.94,12.88,24.90)$ & 18.57 & $(712.91,243.79,310.03)$ & 422.25 & 6.27 \\
ADC(2)-x & $(18.40,13.20,25.65)$ & 19.09 & $(641.26,250.44,314.84)$ & 402.18 & 5.98 \\
ADC(3) & $(17.31,12.68,23.47)$ & 17.82 & $(678.81,281.39,432.19)$ & 464.13 & 7.57 \\
CCSD Der. & $(17.23,12.50,22.58)$ & 17.44 & $(680.32,272.78,384.16)$ & 445.75 & 7.05 \\
CCSD E.V. & $(17.39,12.66,22.98)$ & 17.67 & $(688.15,272.27,388.23)$ & 449.55 & 7.05 \\
\hline
\end{tabular}

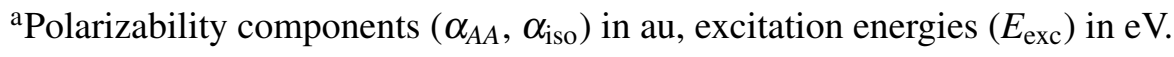


Two more molecules for which experimental data for excited state polarizabilities are available are presented in the following. First, we examine formaldehyde, for which the computational results are displayed in Table III. For all methods, a rather small ground state polarizability is found, which largely increases when the molecules is in the $1^{1} \mathrm{~B}_{1}$ excited state. An approximately 20 -fold increase in isotropic polarizability is present for all computational methods. This is consistent with the experiment, which reports an isotropic polarizability for the ground state as 18.9 au and that of $1^{1} \mathrm{~B}_{1}$ as approximately $410 \pm 180$ au. ${ }^{15,43}$ As such, all computational result are well within the range of the experimentally obtained values. The large increase in polarizability can be rationalized from the Rydberg-type excitation of the state at hand which possesses a large exciton size. This also explains why all components of the polarizability tensor are larger compared to the electronic ground state.

TABLE IV. Static polarizabilities of the naphthalene ground and $1^{1} \mathrm{~B}_{3 \mathrm{u}}$ states. ${ }^{\mathrm{a}}$

\begin{tabular}{l|c|c|c|c|c}
\hline State & \multicolumn{2}{|c|}{ ground state } & \multicolumn{3}{|c}{$1^{1} \mathrm{~B}_{3 \mathrm{u}}$} \\
\hline & $\left(\alpha_{x x}, \alpha_{y y}, \alpha_{z z}\right)$ & $\alpha_{\text {iso }}$ & $\left(\alpha_{x x}, \alpha_{y y}, \alpha_{z z}\right)$ & $\alpha_{\text {iso }}$ & $E_{\text {exc }}$ \\
\hline ADC(2) & $(182.04,133.68,69.29)$ & 128.34 & $(178.48,73.79,44.13)$ & 98.80 & 4.45 \\
ADC(2)-x & $(194.22,140.69,71.34)$ & 135.42 & $(164.97,88.51,50.91)$ & 101.47 & 3.46 \\
ADC(3) & $(177.81,129.95,68.62)$ & 125.46 & $(170.71,98.76,56.14)$ & 108.53 & 4.16 \\
CCSD Der. & $(166.78,123.14,66.67)$ & 118.86 & $(195.12,121.47,70.32)$ & 128.97 & 4.41 \\
CCSD E.V. & $(173.89,128.11,68.22)$ & 123.41 & $(164.30,88.58,54.89)$ & 102.59 & 4.41 \\
Experiment & $(162.0,119.5,70.9)$ & 117.4 & $(186.9,120.1,76.9)$ & 128.0 & 4.02 \\
\hline
\end{tabular}

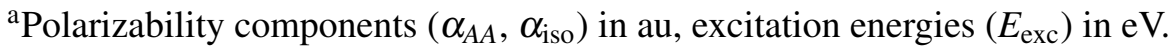

${ }^{\mathrm{b}}$ References 44 and 14.

Next, we consider the ground state and $1{ }^{1} \mathrm{~B}_{3 \mathrm{u}}$ polarizability of naphthalene. The corresponding results from computations and experiment are shown in Table IV. The percentaged deviations from experimental values are depicted in Figure 1. The performance of the computational methods compared to experiment is rather heterogeneous for the ground state polarizability. The largest overestimation for in-plane components $\alpha_{x x}$ and $\alpha_{y y}$ is found for $\operatorname{ADC}(2)$-x with approximately 20\%, whereas CCSD Der. agrees best with the experimental results for these components. Deviations from the experimental $\alpha_{z z}$ result are below 5\%, except for CCSD Der. which underestimates 


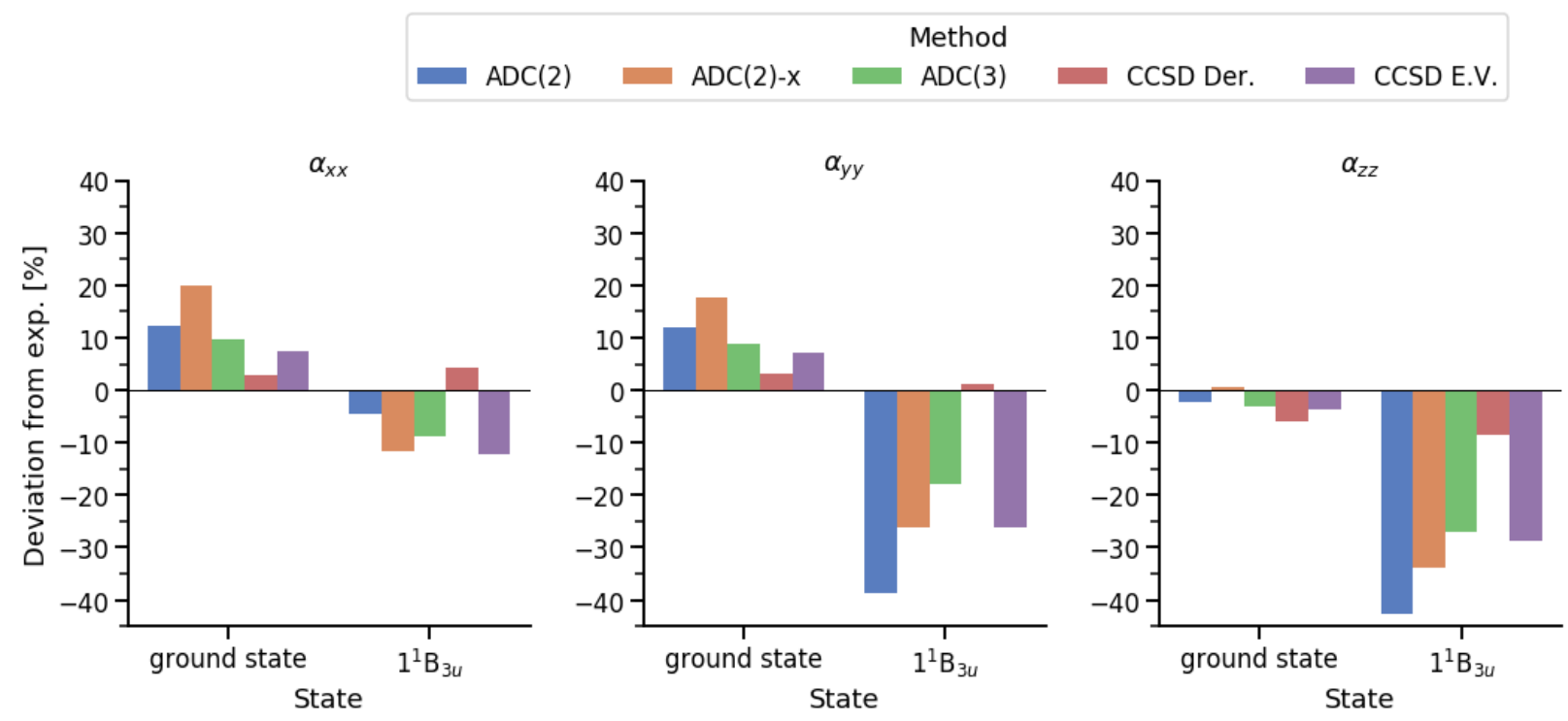

FIG. 1. Deviations of computed polarizability components from the experimental value in percent for the ground and $1^{1} \mathrm{~B}_{3 \mathrm{u}}$ states of naphthalene.

the component by approximately 6\%. As such, all employed methods except for ADC(2)-x yield reliable static polarizabilities for the electronic ground state of naphthalene. In the experiment, a small increase was observed for the static polarizability components of the $1^{1} \mathrm{~B}_{3 \mathrm{u}}$ state compared to the ground state, the largest of which is found for $\alpha_{x x}$. For the polarizabilities based on expectation values, this trend could not be observed in the computational results. Especially the $\alpha_{y y}$ and $\alpha_{z z}$ components are largely underestimated by expectation-value-based methods, the most extreme being $\operatorname{ADC}(2)$ with more than $-40 \%$ deviation for $\alpha_{z z}$. On the contrary, derivative-based EOM-CCSD is capable of describing the trend of small increases in the components correctly. Here, the deviations are below 5\% for $\alpha_{x x}$ and $\alpha_{y y}$, and approximately $-9 \%$ for $\alpha_{z z}$. Thus, one can conclude that in this case amplitude relaxation effects seem to be especially important to model the polarizabilities correctly.

\section{Uracil and $p$-Nitroaniline}

As another example, the ground state and lowest singlet $n \rightarrow \pi^{*}\left(1^{1} A^{\prime \prime}\right)$ and $\pi \rightarrow \pi^{*}\left(1^{1} A^{\prime}\right)$ states of uracil are considered, with results presented in Table V. For the electronic ground state, all five methods again yield comparable results. Similar to $s$-tetrazine and pyrimidine, the $n \rightarrow \pi^{*}$ states have slightly increased polarizabilities for in-plane components $\alpha_{x x}$ and $\alpha_{y y}$, when derivative- 
Complex Excited State Polarizabilities using ADC

TABLE V. Static polarizabilities of the uracil ground state, $1^{1} \mathrm{~A}^{\prime \prime}$, and $2^{1} \mathrm{~A}^{\prime} .{ }^{\mathrm{a}}$

\begin{tabular}{l|c|c|c|c|c|c|c|c}
\hline State & \multicolumn{2}{|c|}{ ground state } & \multicolumn{2}{|c|}{$1^{1} \mathrm{~A}^{\prime \prime}$} & \multicolumn{2}{|c}{$2^{1} \mathrm{~A}^{\prime}$} \\
& $\left(\alpha_{x x}, \alpha_{y y}, \alpha_{z z}\right)$ & $\alpha_{\text {iso }}$ & $\left(\alpha_{x x}, \alpha_{y y}, \alpha_{z z}\right)$ & $\alpha_{\text {iso }}$ & $E_{\text {exc }}$ & $\left(\alpha_{x x}, \alpha_{y y}, \alpha_{z z}\right)$ & $\alpha_{\text {iso }}$ & $E_{\text {exc }}$ \\
\hline ADC(2) & $(105.23,80.00$, & 76.20 & $(86.44,67.30$, & 60.28 & 4.73 & $(138.88,81.77$, & 85.37 & 5.32 \\
& $43.37)$ & & $27.08)$ & & & $35.46)$ & & \\
ADC(2)-x & $(110.75,83.17$, & 79.47 & $(85.17,73.16$, & 63.83 & 3.97 & $(147.08,87.72$, & 91.06 & 4.61 \\
& $44.48)$ & & $33.17)$ & & & $38.37)$ & & \\
ADC(3) & $(98.47,76.27$, & 72.35 & $(74.72,70.74$, & 60.50 & 5.36 & $(105.12,86.93$, & 78.11 & 5.38 \\
& $42.30)$ & & $36.03)$ & & & $42.27)$ & & \\
CCSD Der. & $(95.82,74.62$, & 70.67 & $(98.08,88.29$, & 77.19 & 5.22 & $(133.94,102.78$, & 96.14 & 5.58 \\
& $41.59)$ & & $45.19)$ & & & $51.69)$ & & \\
CCSD E.V. & $(98.75,76.41$, & 72.53 & $(71.49,69.43$, & 58.49 & 5.22 & $(112.82,85.86$, & 80.55 & 5.58 \\
& $42.42)$ & & $34.55)$ & & & $42.97)$ & & \\
\hline
\end{tabular}

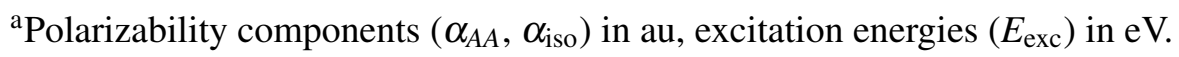

based EOM-CCSD is used. This is not the case for the expectation-value methods. In fact, all ADC and the EOM-CCSD E.V. results show a noticeable drop in polarizability for this state of uracil. Surprisingly, this discrepancy is not reduced when employing ADC(3), but instead becomes even larger. EOM-CCSD E.V. here yields values similar to ADC(3). For the $\pi \rightarrow \pi^{*}$ transition, both dipole moments and polarizabilities show a large increase. ${ }^{12}$ For this state the $\alpha_{x x}$ component of the polarizability increases the most, due to the large coupling matrix element to the ground state. Again, ADC(3) and EOM-CCSD E.V. show a much smaller increase for this component than EOM-CCSD Der.

Results for PNA are depicted in Table VI, including polarizability components of the electronic ground state and the lowest singlet excited $\pi \rightarrow \pi^{*}$ state $\left(2^{1} \mathrm{~A}_{1}\right)$. Ground state polarizabilities are again similar. The probed singlet state corresponds to a strong intramolecular charge-transfer (CT) excitation. ${ }^{12}$ As such, the corresponding dipole moment increases upon excitation, yielding a species with more ionic character than in the ground state. The excitation still shows a large oscillator strength, i.e., transition dipole moment along the $z$-axis. Therefore, the polarizability of the $1^{1} A_{1}$ largely increases in the $\alpha_{z z}$ component for all presented methods - particularly using 
TABLE VI. Static polarizabilities of the PNA ground state and $2^{1} \mathrm{~A}_{1} \cdot{ }^{\mathrm{a}}$

\begin{tabular}{l|c|c|c|c|c}
\hline State & \multicolumn{2}{|c|}{ ground state } & \multicolumn{2}{c}{$2^{1} \mathrm{~A}_{1}$} & \multicolumn{2}{l}{} \\
\hline & $\left(\alpha_{x x}, \alpha_{y y}, \alpha_{z z}\right)$ & $\alpha_{\text {iso }}$ & $\left(\alpha_{x x}, \alpha_{y y}, \alpha_{z z}\right)$ & $\alpha_{\text {iso }}$ & $E_{\text {exc }}$ \\
\hline ADC(2) & $(118.84,58.94,168.68)$ & 115.49 & $(68.26,49.52,196.97)$ & 104.92 & 4.30 \\
ADC(2)-x & $(125.29,60.62,183.45)$ & 123.12 & $(82.44,50.60,176.49)$ & 103.18 & 3.56 \\
ADC(3) & $(112.76,58.02,162.94)$ & 111.24 & $(86.53,59.25,204.37)$ & 116.72 & 4.23 \\
CCSD Der. & $(106.38,56.95,152.90)$ & 105.41 & $(109.60,83.60,239.08)$ & 144.09 & 4.62 \\
CCSD E.V. & $(110.76,58.20,157.68)$ & 108.88 & $(74.95,69.30,200.89)$ & 115.05 & 4.62 \\
\hline
\end{tabular}

a Polarizability components $\left(\alpha_{A A}, \alpha_{\text {iso }}\right)$ in au, excitation energies $\left(E_{\text {exc }}\right)$ in $\mathrm{eV}$.

CCSD Der. ADC(3) and EOM-CCSD E.V. behave similarly for the excited state, with a deviation $\delta_{\text {Der. }}$ to EOM-CCSD Der. of 27.37 au and 29.04 au, respectively. Discrepancies of ADC(2) and ADC(2)-x are larger by approximately $10 \mathrm{au}$, amounting to 39.17 au and $40.91 \mathrm{au}$, respectively. Hence, the $\pi \rightarrow \pi^{*}$ intramolecular CT state shows the largest differences between derivative- and expectation-value-based methods studied here. The effects of full amplitude response in case of a CT excitation seem to have a large impact on the excited state polarizability of the respective state. As such, care should be taken in these cases. Nevertheless, ADC methods are capable to capture the trend of an increasing $\alpha_{z z}$ component for CT excitations correctly, whereas methods like time-dependent DFT tend to fail in this case. ${ }^{45,46}$

To summarize this brief study of static excited state polarizabilities, our presented findings both match the expected trends and previously published results, suggesting that our implementation is comparable to related methodologies using an expectation-value-based ansatz. We have shown that the agreement between methods solely depends on the approach to evaluate the polarizability, and not whether ADC or CC is chosen as underlying quantum chemical method. Hence, it would also be interesting to see how derivative-based ADC excited state polarizabilities would compare to derivative-based EOM-CCSD. In this case, amplitude-relaxed second derivatives of the ADC excited state energy would need to be derived and implemented, which is beyond the scope of this work. In addition, note that the ISR-based ansatz requires much less computational effort, yielding excited state polarizabilities for the price of ground state polarizabilities, once the excited states are determined. In a derivative-based approach, however, more response equations need to 
be solved..$^{12}$

\section{D. $C_{6}$ Dispersion Coefficients for Excited States}

Until now, we have only considered static polarizabilities of excited states, which do not require solutions of the complex response function. For $C_{6}$ dispersion coefficients, however, the isotropic average of the molecular dipole polarizability as a function of purely imaginary frequencies is needed to compute the interaction between two systems through the so-called Casimir-Polder potential. ${ }^{6,47}$ With the Python function to solve eq (8) in place, the required Gauss-Legendre integration can be easily carried out using built-in NumPy functions, ${ }^{48}$ as shown in the code snippet below (Fig. 2). This code example shows again how well adcc integrates with the Python ecosys-

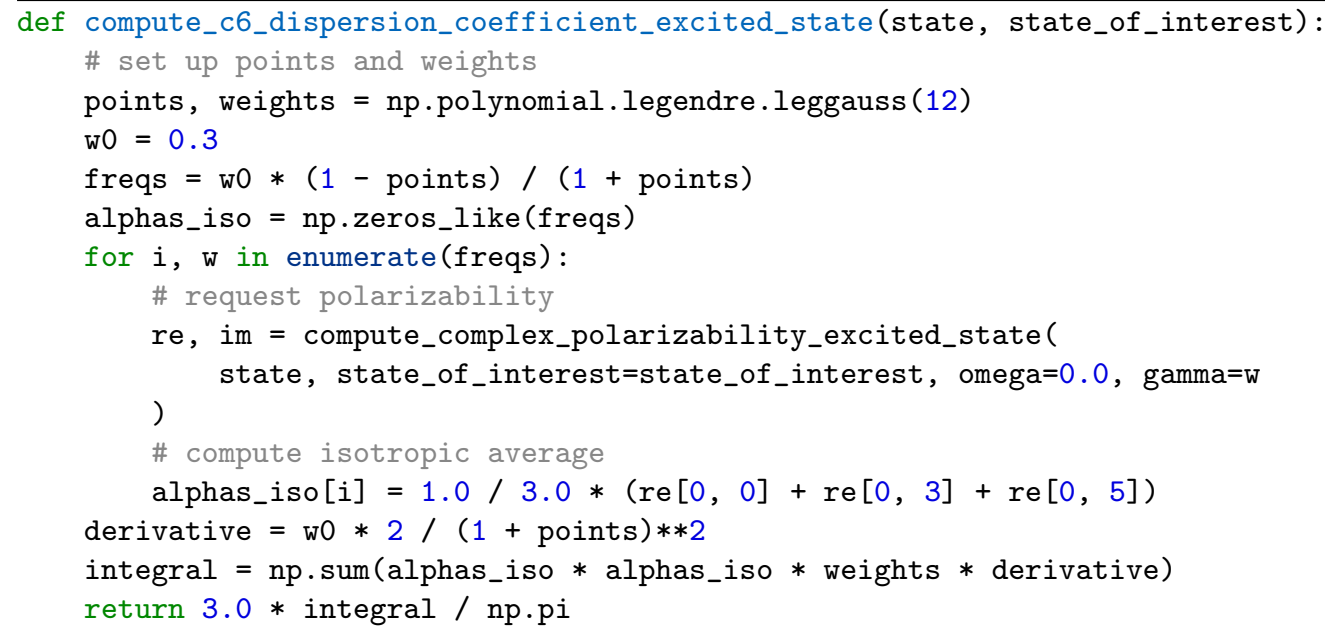

FIG. 2. Python function to compute the $C_{6}$ dispersion coefficients with adcc.

tem, making it possible to quickly implement new features with only minor effort. In addition, the rich feature set of NumPy makes it possible to write code that strongly resembles the text book equations.

To illustrate the above routine, we computed $C_{6}$ dispersion coefficients for the excited states of our previously studied molecules, using $\mathrm{ADC}(2), \mathrm{ADC}(2)-\mathrm{x}$, and $\mathrm{ADC}(3)$ for $s$-tetrazine and pyrimidine, and ADC(2) only for uracil and PNA. The results are shown in Table VII. Since no experimental or computational results from a similar method are, to the best of our knowledge, available for excited state dispersion coefficients, our here presented discussion of the computed values unfortunately remains scarce, and can only serve as a proof of principle. Nonetheless, we 
TABLE VII. $C_{6}$ dispersion coefficients of electronic ground and excited states employing ADC

\begin{tabular}{l|l|lll}
\hline \multirow{2}{*}{ System } & State & \multicolumn{3}{|c}{$C_{6}$ dispersion coefficient [au] } \\
& & ADC(2) & ADC(2)-x & ADC(3) \\
\hline s-tetrazine & ground state & 1161.05 & 1197.54 & 1129.03 \\
& $1{ }^{1} \mathrm{~B}_{1 \mathrm{u}}$ & 642.85 & 795.37 & 910.02 \\
\hline pyrimidine & ground state & 1510.36 & 1555.60 & 1468.87 \\
\hline uracil & $1{ }^{1} \mathrm{~B}_{2}$ & 889.05 & 1044.24 & 1160.84 \\
& ground state & 2237.45 & $\ldots$ & $\ldots$ \\
\hline PNA & $1{ }^{1} \mathrm{~A}^{\prime \prime}$ & 1099.27 & $\ldots$ & $\ldots$ \\
& $2^{1} \mathrm{~A}^{\prime}$ & 1355.94 & $\ldots$ & $\ldots$ \\
\hline & ground state & 4396.76 & $\ldots$ & $\ldots$ \\
\hline
\end{tabular}

emphasize the methodological and implementation aspect of our work, which makes it possible to easily evaluate complex response functions of electronically excited states. Our Python-driven toolkit adcc offers the ideal platform to experiment with such more "exotic" response properties.

\section{CONCLUSIONS}

We have presented the first derivation and implementation of complex, frequency-dependent excited state polarizabilities for ADC using an ISR-based ansatz. The derivation elegantly illustrates how ADC/ISR response properties for excited state can be treated in general. The presented derivation further demonstrates how the ADC/ISR framework, in combination with the adcc toolkit, can be used to perform rapid prototyping of new response property functionalities. Given the fact that only ground state response properties were derived with ADC so far, our presented theoretical methodology serves as a blueprint paving the way for arbitrary response functions with ADC/ISR. The shown derivation will for example serve as a guideline to implement more excited state response properties, for example the process of resonant two-photon absorption. To verify that the implemented methodology is consistent with similar methodologies, we have presented calculations of static excited state polarizabilities in comparison with EOM-CCSD. The general formulation and implementation in adcc also allows to evaluate the respective re- 
sponse property for open-shell molecules on top of an unrestricted Hartree-Fock reference, or for

systems with few-reference character using the spin-flip ADC ansatz. ${ }^{49}$ From a practical point of view, our method could serve as a low-cost $a b$-initio method to benchmark excited state polarizabilities and dispersion coefficients obtained from DFT-based methologies. These are for example needed to parametrize force fields for classical molecular dynamics simulations of excited states. ${ }^{50}$ Performing atomistic decompositions of our derived properties is, however, beyond the scope of this article. We hope that our presented methodology will serve as a useful template for similar response properties in future work.

\section{DATA AVAILABILITY STATEMENT}

The data that support the findings of this study are openly available on GitHub (https:// github.com/maxscheurer/adc_excipol_data) and have been deposited on Zenodo with the DOI $10.5281 /$ zenodo. 3770508.

\section{ACKNOWLEDGMENTS}

MS is supported by the DFG by means of the research training group "CLiC" (GRK 1986, Complex Light Control) and by the Heidelberg Graduate School of Mathematical and Computational Methods for the Sciences (GSC220). TF and PN acknowledge financial support from the Swedish Research Council (Grants No. 2017-00356 and 2018-04343). The authors thank M. Hodecker for helpful discussions.

\section{Appendix A: Matrix-Vector Product of a second-order ISR One-Particle Operator}

Based on the original equations from Trofimov and Schirmer ${ }^{4}$, we derived the matrix-vector product of the ISR one-particle operator with a vector $\mathbf{v}$ through second order in perturbation theory, i.e., $\mathbf{B v}=\mathbf{r}$. The $\mathbf{B}$ matrix possesses a block structure similar to that of the ADC matrix, ${ }^{4}$ such that the vectors $\mathbf{v}$ and $\mathbf{r}$ contain a singles and a doubles block. In the following, the indices $i, j, k, l, m, n$ refer to occupied molecular orbitals, $a, b, c, d, e, f$ refer to virtual ones, and $p, q, r, s$ are general molecular orbital indices. Within the equations, anti-symmetrized two-electron integrals 
Complex Excited State Polarizabilities using ADC

$\langle p q \| r q\rangle$ occur, together with the T2-amplitudes defined as

$$
t_{i j}^{a b}=\frac{\langle i j \| a b\rangle}{\varepsilon_{a}+\varepsilon_{b}-\varepsilon_{i}-\varepsilon_{j}},
$$

where $\varepsilon_{p}$ denotes the energy of HF orbital $p$. Furthermore, the MP(2) density matrix contribution ${ }^{51}$ is defined as

$$
\rho_{i a}^{(2)}=-\frac{1}{2\left(\varepsilon_{a}-\varepsilon_{i}\right)}\left[\sum_{j b c} t_{i j}^{b c}\langle j a \| b c\rangle+\sum_{j k b} t_{j k}^{a b}\langle j k \| i b\rangle\right] .
$$

The permutation operator $\hat{\mathcal{P}}_{p r q q}$ permutes the index pairs $(p, q)$ and $(r, s)$. Collecting all terms, one finds the result for the singles block of the matrix-vector product as

$$
\begin{aligned}
r_{a i}= & \sum_{c} d_{a c} v_{c i}-\sum_{k} d_{i k} v_{a k}-\sum_{c} v_{c i}\left(\sum_{j} \rho_{j a}^{(2)} d_{c j}+\rho_{j c}^{(2)} d_{a j}\right)-\sum_{k} v_{a k}\left(\sum_{b} \rho_{i b}^{(2)} d_{b k}+\rho_{k b}^{(2)} d_{b i}\right) \\
& -\frac{1}{4} \sum_{c} v_{c i}\left(\sum_{e f m n} t_{m n}^{e f}\left(t_{m n}^{a f} d_{e c}+t_{m n}^{c f} d_{e a}\right)\right)+\sum_{c} v_{c i}\left(-\frac{1}{2} \sum_{e f m n} t_{m n}^{c e} t_{m n}^{a f} d_{e f}+\sum_{f m n j} t_{m n}^{c f} t_{j n}^{a f} d_{j m}\right) \\
& +\frac{1}{4} \sum_{k} v_{a k}\left(\sum_{e f m n} t_{m n}^{e f}\left(t_{i n}^{e f} d_{k m}+t_{k n}^{e f} d_{i m}\right)\right)+\sum_{k} v_{a k}\left(-\sum_{e d f n} t_{k n}^{e f} t_{i n}^{d f} d_{e d}+\frac{1}{2} \sum_{e f m n} t_{k n}^{e f} t_{i m}^{e f} d_{m n}\right) \\
& +\frac{1}{2} \sum_{c k} v_{c k}\left(\left(1+\hat{\mathcal{P}}_{a i, c k}\right)\left(\sum_{e f n} t_{k n}^{e f} t_{i n}^{a f} d_{e c}-\sum_{f m n} t_{m n}^{c f} t_{i n}^{a f} d_{k m}\right)\right)+\sum_{c k} v_{c k}\left(-\sum_{f m n} t_{k n}^{c f} t_{i m}^{a f} d_{m n}+\sum_{e f n} t_{k n}^{c e} t_{i n}^{a f} d_{e f}\right) \\
& -2 \sum_{d l} v_{a d i l}\left(d_{l d}-\sum_{f n} t_{l n}^{d f} d_{f n}\right)+2 \sum_{c l} v_{c a i l}\left(d_{l c}-\sum_{f n} t_{l n}^{c f} d_{f n}\right) \\
& -2 \sum_{d k l} v_{a d k l} \sum_{e} t_{k l}^{e d} d_{e i}-2 \sum_{c d l} v_{c d i l} \sum_{n} t_{n l}^{c d} d_{a n} .
\end{aligned}
$$

The doubles part of the vector is given by

$$
\begin{aligned}
r_{a b i j}= & -v_{a i}\left(d_{j b}-\sum_{f n} t_{j n}^{b f} d_{f n}\right)+v_{a j}\left(d_{i b}-\sum_{f n} t_{i n}^{b f} d_{f n}\right)+v_{b i}\left(d_{j a}-\sum_{f n} t_{j n}^{a f} d_{f n}\right)-v_{b j}\left(d_{i a}-\sum_{f n} t_{i n}^{a f} d_{f n}\right) \\
& -\sum_{k} v_{a k} \sum_{e} t_{i j}^{e b} d_{e k}+\sum_{k} v_{b k} \sum_{e} t_{i j}^{e a} d_{e k}-\sum_{c} v_{c i} \sum_{n} t_{n j}^{a b} d_{c n}+\sum_{c} v_{c j} \sum_{n} t_{n i}^{a b} d_{c n} \\
& +2 \sum_{c} d_{a c} v_{c b i j}-d_{b c} v_{c a i j}-2 \sum_{k} d_{k i} v_{a b k j}-d_{k j} v_{a b k i} .
\end{aligned}
$$

\section{REFERENCES}

${ }^{1}$ T. Helgaker, S. Coriani, P. Jørgensen, K. Kristensen, J. Olsen, and K. Ruud, "Recent advances in wave function-based methods of molecular-property calculations," Chem. Rev. 112, 543-631 (2012). 
${ }^{2}$ P. Norman, K. Ruud, and T. Saue, Principles and Practices of Molecular Properties; Theory, Modeling and Simulations (John Wiley \& Sons, 2018).

${ }^{3}$ J. Schirmer, "Beyond the random-phase approximation: A new approximation scheme for the polarization propagator,' Phys. Rev. A 26, 2395-2416 (1982).

${ }^{4}$ J. Schirmer and A. B. Trofimov, "Intermediate state representation approach to physical properties of electronically excited molecules," J. Chem. Phys. 120, 11449-11464 (2004).

${ }^{5}$ A. B. Trofimov, I. L. Krivdina, J. Weller, and J. Schirmer, “Algebraic-diagrammatic construction propagator approach to molecular response properties," Chem. Phys. 329, 1-10 (2006).

${ }^{6}$ T. Fransson, D. R. Rehn, A. Dreuw, and P. Norman, "Static polarizabilities and $\mathrm{C}_{6}$ dispersion coefficients using the algebraic-diagrammatic construction scheme for the complex polarization propagator,” J. Chem. Phys. 146, 094301 (2017).

${ }^{7}$ M. Hodecker, D. R. Rehn, P. Norman, and A. Dreuw, "Algebraic-diagrammatic construction scheme for the polarization propagator including ground-state coupled-cluster amplitudes. ii. static polarizabilities,” J. Chem. Phys. 150, 174105 (2019).

${ }^{8}$ S. Knippenberg, D. R. Rehn, M. Wormit, J. H. Starcke, I. L. Rusakova, A. B. Trofimov, and A. Dreuw, "Calculation of nonlinear response properties using the intermediate state representation and the algebraic-diagrammatic construction polarization propagator approach: Two-photon absorption spectra,” J. Chem. Phys. 136, 064107 (2012).

${ }^{9}$ D. R. Rehn, A. Dreuw, and P. Norman, "Resonant inelastic x-ray scattering amplitudes and cross section in the algebraic diagrammatic construction/intermediate state representation (ADC/ISR) approach,” J. Chem. Theory Comput. 13, 5552-5559 (2017).

${ }^{10}$ D. Jonsson, P. Norman, Y. Luo, and H. Ågren, "Response theory for static and dynamic polarizabilities of excited states," J. Chem. Phys. 105, 581 (1996).

${ }^{11}$ C. Hättig, O. Christiansen, S. Coriani, and P. Jørgensen, “Static and frequency-dependent polarizabilities of excited singlet states using coupled cluster response theory," J. Chem. Phys. 109, 9237-9243 (1998).

${ }^{12}$ K. D. Nanda and A. I. Krylov, "Static polarizabilities for excited states within the spinconserving and spin-flipping equation-of-motion coupled-cluster singles and doubles formalism: Theory, implementation, and benchmarks," J. Chem. Phys. 145, 204116 (2016).

${ }^{13}$ M. F. Herbst, M. Scheurer, T. Fransson, D. R. Rehn, and A. Dreuw, "adcc: A versatile toolkit for rapid development of algebraic-diagrammatic construction methods," (2020). 
${ }^{14}$ P. Norman, D. Jonsson, and H. Ågren, "Excited state properties through cubic response theory: polarizabilities of benzene and naphthalene," Chem. Phys. Lett. 268, 337-344 (1997).

${ }^{15}$ D. Jonsson, P. Norman, and H. Ågren, "Single determinant calculations of excited state polarizabilities," Chem. Phys. 224, 201-214 (1997).

${ }^{16}$ D. Jonsson, P. Norman, H. Ågren, Y. Luo, K. O. Sylvester-Hvid, and K. V. Mikkelsen, “Excited state polarizabilities in solution obtained by cubic response theory: Calculations on para-, ortho-, and meta-nitroaniline," J. Chem. Phys. 109, 6351 (1998).

${ }^{17}$ J. F. Stanton and J. Gauss, "The first excited singlet state of s -tetrazine: A theoretical analysis of some outstanding questions," J. Chem. Phys. 104, 9859-9869 (1996).

${ }^{18}$ L. F. Pasteka, M. Melichercik, P. Neogrady, and M. Urban, "CASPT2 and CCSD(T) calculations of dipole moments and polarizabilities of acetone in excited states," Mol. Phys. 110, 2219-2237 (2012).

${ }^{19}$ N. K. Graf, D. H. Friese, N. O. C. Winter, and C. Hättig, "Excited state polarizabilities for CC2 using the resolution-of-the-identity approximation,” J. Chem. Phys. 143, 244108 (2015).

${ }^{20}$ M. Medved, S. Budzak, and T. Pluta, "Electric properties of the low-lying excited states of benzonitrile: geometry relaxation and solvent effects," Theor. Chem. Acc. 134, 78 (2015).

${ }^{21}$ K. D. Nanda and A. I. Krylov, "Effect of the diradical character on static polarizabilities and two-photon absorption cross sections: A closer look with spin-flip equation-of-motion coupledcluster singles and doubles method,” J. Chem. Phys. 146, 224103 (2017).

${ }^{22}$ M. Schütz, J. Hutter, and H. P. Lüthi, “The molecular and electronic structure of s-tetrazine in the ground and first excited state: A theoretical investigation,” J. Chem. Phys. 103, 7048 (1995).

${ }^{23}$ B. Jansik, D. Jonsson, P. Salek, and H. Ågren, “Calculations of static and dynamic polarizabilities of excited states by means of density functional theory," J. Chem. Phys. 121, 7595-7600 (2004).

${ }^{24}$ P. Norman, A. Jiemchooroj, and B. E. Sernelius, "First principle calculations of dipole-dipole dispersion coefficients for the ground and first $\pi \rightarrow \pi^{*}$ excited states of some azabenzenes," J. Comp. Methods Sci. Eng. 4, 321-332 (2004).

${ }^{25}$ R. F. Pauszek III, G. Kodali, and R. J. Stanley, "Excited state electronic structures of 5,10-methenyltetrahydrofolate and 5,10-methylenetetrahydrofolate determined by Stark spectroscopy," J. Phys. Chem. A 118, 8320-8328 (2014).

${ }^{26}$ M. Hodecker, D. R. Rehn, A. Dreuw, and S. Höfener, "Similarities and differences of the lagrange formalism and the intermediate state representation in the treatment of molecular prop- 
erties," J. Chem. Phys. 150, 164125 (2019).

${ }^{27}$ J. F. Stanton and R. J. Bartlett, "The equation of motion coupled-cluster method. A systematic biorthogonal approach to molecular excitation energies, transition probabilities, and excited state properties," J. Chem. Phys. 98, 7029 (1993).

${ }^{28}$ K. D. Nanda and A. I. Krylov, “Two-photon absorption cross sections within equation-of-motion coupled-cluster formalism using resolution-of-the-identity and cholesky decomposition representations: Theory, implementation, and benchmarks,” J. Chem. Phys. 142, 064118 (2015).

${ }^{29}$ A. Dreuw and M. Wormit, "The algebraic diagrammatic construction scheme for the polarization propagator for the calculation of excited states," WIREs Comput. Mol. Sci. 5, 82-95 (2015).

${ }^{30}$ J. Schirmer, Many-Body Methods for Atoms, Molecules and Clusters (Springer, 2018).

${ }^{31}$ M. R. Hestenes and E. Stiefel, "Methods of conjugate gradients for solving linear systems," J. Res. Natl. Bur. Stand. 49, 409-436 (1952).

${ }^{32}$ H. Walker and P. Ni, “Anderson acceleration for fixed-point iterations," SINUM 49, 1715-1735 (2011).

${ }^{33}$ A. J. Sadlej, Colect. Czech. Chem. Commun. 53, 1995-2016 (1988).

${ }^{34}$ Q. Sun, T. C. Berkelbach, N. S. Blunt, G. H. Booth, S. Guo, Z. Li, J. Liu, J. McClain, E. R. Sayfutyarova, S. Sharma, S. Wouters, and G. K.-L. Chan, "The python-based simulations of chemistry framework (pyscf),” WIREs Comput. Mol. Sci. 8 (2017), 10.1002/wcms.1340.

${ }^{35}$ Q. Sun, X. Zhang, S. Banerjee, P. Bao, M. Barbry, N. S. Blunt, N. A. Bogdanov, G. H. Booth, J. Chen, Z.-H. Cui, J. J. Eriksen, Y. Gao, S. Guo, J. Hermann, M. R. Hermes, K. Koh, P. Koval, S. Lehtola, Z. Li, J. Liu, N. Mardirossian, J. D. McClain, M. Motta, B. Mussard, H. Q. Pham, A. Pulkin, W. Purwanto, P. J. Robinson, E. Ronca, E. Sayfutyarova, M. Scheurer, H. F. Schurkus, J. E. T. Smith, C. Sun, S.-N. Sun, S. Upadhyay, L. K. Wagner, X. Wang, A. White, J. D. Whitfield, M. J. Williamson, S. Wouters, J. Yang, J. M. Yu, T. Zhu, T. C. Berkelbach, S. Sharma, A. Sokolov, and G. K.-L. Chan, "Recent developments in the pyscf program package," (2020), arXiv:2002.12531.

${ }^{36}$ Y. Shao, Z. Gan, E. Epifanovsky, A. T. Gilbert, M. Wormit, J. Kussmann, A. W. Lange, A. Behn, J. Deng, X. Feng, D. Ghosh, M. Goldey, P. R. Horn, L. D. Jacobson, I. Kaliman, R. Z. Khaliullin, T. Kuś, A. Landau, J. Liu, E. I. Proynov, Y. M. Rhee, R. M. Richard, M. A. Rohrdanz, R. P. Steele, E. J. Sundstrom, H. L. Woodcock, P. M. Zimmerman, D. Zuev, B. Albrecht, E. Alguire, B. Austin, G. J. O. Beran, Y. A. Bernard, E. Berquist, K. Brandhorst, K. B. Bravaya, S. T. Brown, D. Casanova, C.-M. Chang, Y. Chen, S. H. Chien, K. D. Closser, D. L. Crittenden, 
M. Diedenhofen, R. A. DiStasio, H. Do, A. D. Dutoi, R. G. Edgar, S. Fatehi, L. Fusti-Molnar, A. Ghysels, A. Golubeva-Zadorozhnaya, J. Gomes, M. W. Hanson-Heine, P. H. Harbach, A. W. Hauser, E. G. Hohenstein, Z. C. Holden, T.-C. Jagau, H. Ji, B. Kaduk, K. Khistyaev, J. Kim, J. Kim, R. A. King, P. Klunzinger, D. Kosenkov, T. Kowalczyk, C. M. Krauter, K. U. Lao, A. D. Laurent, K. V. Lawler, S. V. Levchenko, C. Y. Lin, F. Liu, E. Livshits, R. C. Lochan, A. Luenser, P. Manohar, S. F. Manzer, S.-P. Mao, N. Mardirossian, A. V. Marenich, S. A. Maurer, N. J. Mayhall, E. Neuscamman, C. M. Oana, R. Olivares-Amaya, D. P. O’Neill, J. A. Parkhill, T. M. Perrine, R. Peverati, A. Prociuk, D. R. Rehn, E. Rosta, N. J. Russ, S. M. Sharada, S. Sharma, D. W. Small, A. Sodt, T. Stein, D. Stück, Y.-C. Su, A. J. Thom, T. Tsuchimochi, V. Vanovschi, L. Vogt, O. Vydrov, T. Wang, M. A. Watson, J. Wenzel, A. White, C. F. Williams, J. Yang, S. Yeganeh, S. R. Yost, Z.-Q. You, I. Y. Zhang, X. Zhang, Y. Zhao, B. R. Brooks, G. K. Chan, D. M. Chipman, C. J. Cramer, W. A. Goddard, M. S. Gordon, W. J. Hehre, A. Klamt, H. F. Schaefer, M. W. Schmidt, C. D. Sherrill, D. G. Truhlar, A. Warshel, X. Xu, A. Aspuru-Guzik, R. Baer, A. T. Bell, N. A. Besley, J.-D. Chai, A. Dreuw, B. D. Dunietz, T. R. Furlani, S. R. Gwaltney, C.-P. Hsu, Y. Jung, J. Kong, D. S. Lambrecht, W. Liang, C. Ochsenfeld, V. A. Rassolov, L. V. Slipchenko, J. E. Subotnik, T. Van Voorhis, J. M. Herbert, A. I. Krylov, P. M. Gill, and M. Head-Gordon, "Advances in molecular quantum chemistry contained in the Q-Chem 4 program package," Mol. Phys. 113, 184-215 (2015).

${ }^{37}$ N. M. O’Boyle, A. L. Tenderholt, and K. M. Langner, “cclib: A Library for PackageIndependant Computational Chemistry Algorithms," J. Comput. Chem. 29, 839-845 (2008), NIHMS150003.

${ }^{38}$ W. McKinney, "Data Structures for Statistical Computing in Python," in Proc. 9th Python Sci. Conf., edited by S. van der Walt and J. Millman (2010) pp. 51-56.

${ }^{39}$ W. McKinney, "pandas: a Foundational Python Library for Data Analysis and Statistics," (2011).

${ }^{40}$ J. D. Hunter, “Matplotlib: A 2D Graphics Environment," Comput. Sci. Eng. 9, 99-104 (2007), arXiv:0402594v3 [arXiv:cond-mat].

${ }^{41}$ M. Waskom, O. Botvinnik, J. Ostblom, S. Lukauskas, P. Hobson, MaozGelbart, D. C. Gemperline, T. Augspurger, Y. Halchenko, J. B. Cole, J. Warmenhoven, J. de Ruiter, C. Pye, S. Hoyer, J. Vanderplas, S. Villalba, G. Kunter, E. Quintero, P. Bachant, M. Martin, K. Meyer, C. Swain, A. Miles, T. Brunner, D. O’Kane, T. Yarkoni, M. L. Williams, and C. Evans, “mwaskom/seaborn: v0.10.0," (2020). 
${ }^{42}$ S. Heitz, D. Weidauer, and A. Hese, "Measurement of static polarizabilities on s-tetrazine," J. Chem. Phys. 95, 7952-7956 (1991).

${ }^{43}$ E. C. Hurdis and C. P. Smyth, "The structural effects of unsaturation and hyperconjugation in aldehydes, nitriles and chlorides as shown by their dipole moments in the vapor state," J. Am. Chem. Soc. 65, 89-96 (1943).

${ }^{44}$ S. Heitz, D. Weidauer, B. Rosenow, and A. Hese, "Measurement of static polarizabilities on C10H8 and C10D8," J. Chem. Phys. 96, 976-981 (1992).

${ }^{45}$ A. Dreuw and M. Head-Gordon, "Failure of Time-Dependent Density Functional Theory for Long-Range Charge-Transfer Excited States: The Zincbacteriochlorin-Bacteriochlorin and Bacteriochlorophyll-Spheroidene Complexes,” J. Am. Chem. Soc. 126, 4007-4016 (2004).

${ }^{46}$ J. J. Eriksen, S. P. Sauer, K. V. Mikkelsen, O. Christiansen, H. J. A. Jensen, and J. Kongsted, "Failures of TDDFT in describing the lowest intramolecular charge-transfer excitation in paranitroaniline," Mol. Phys. 111, 1235-1248 (2013).

${ }^{47}$ H. B. G. Casimir and D. Polder, "The influence of retardation on the London-van der Waals forces," Phys. Rev. 73, 360-372 (1948).

${ }^{48}$ S. Van Der Walt, S. C. Colbert, and G. Varoquaux, "The NumPy array: A structure for efficient numerical computation,” Comput. Sci. Eng. 13, 22-30 (2011), arXiv:1102.1523.

${ }^{49}$ D. Lefrancois, M. Wormit, and A. Dreuw, "Adapting algebraic diagrammatic construction schemes for the polarization propagator to problems with multi-reference electronic ground states exploiting the spin-flip ansatz," J. Chem. Phys. 143, 124107 (2015), https://doi.org/10.1063/1.4931653.

${ }^{50}$ E. Heid, P. A. Hunt, and C. Schröder, "Evaluating excited state atomic polarizabilities of chromophores," Phys. Chem. Chem. Phys. 20, 8554-8563 (2018).

${ }^{51}$ M. Wormit, D. R. Rehn, P. H. Harbach, J. Wenzel, C. M. Krauter, E. Epifanovsky, and A. Dreuw, "Investigating excited electronic states using the algrebraic diagrammatic construction (ADC) approach for the polarisation propagator," Mol. Phys. 112, 774 (2014). 